\title{
Ovarian torsion- visualized with MR imaging
}

\author{
Julia Röper-Kelmayr ${ }^{1 *}$, Christian Asel ${ }^{1}$ and Franz A. Fellner ${ }^{1,2}$ \\ ${ }^{1}$ Central Radiology Institute, Kepler University Hospital, Medical Faculty of the Johannes Kepler University, Linz, Austria \\ ${ }^{2}$ Medical Faculty of the Friedrich-Alexander-University of Erlangen-Nürnberg, Erlangen, Germany
}

\section{Case report}

A 15-year-old female came into our hospital's emergency room complaining of right- sided lower abdominal pain. There has been a former event of ovarian torsion two years ago, which had led to surgery at that time.

Now, primarily performed ultrasound was not fully conclusive. Therefore, and due to resistance of therapy, the patient underwent immediately MR imaging of the abdomen.

The images revealed a significantly distended right ovary with increased hyperintense signals of parenchyma in T2- weighted scans, as well as poor contrast enhancement in T1- post contrast- scans. Furthermore, the scans revealed right- sided adnexal torsion of 360 degrees and free intra- abdominal fluid.

Immediately after the MR examination, the patient underwent surgical therapy of the ovarian torsion.

\section{Discussion}

Ovarian torsion is a possible cause of acute abdominal pain in women at any age, especially during their reproductive years $[1,2]$. Ultrasound imaging, even if using color coded Doppler technique may be inconclusive, though an enlarged ovary in association with "whirlpool- sign" is diagnostic [3]. Notwithstanding, that there are hints that can lead to correct diagnosis in CT [1], an MR examination should be preferred in young patients.

An unilateral enlarged ovary does not prove an existing torsion. There are ovarian tumors, metastases, ectopic pregnancy or hemorrhagic cysts to consider. In case of ovarian torsion there is usually additionally edematous swelling of the affected ovary with peripheral located follicles as a result of vascular congestion. This can be distinguished preeminently by means of magnetic resonance imaging.

To verify a twisted pedicle, multiplanar acquisitions, especially coronal sequences, are helpful for detection of this disorder. Furthermore, absence of contrast enhancement of the ovarian parenchyma in T1-weighted post contrast- scans indicate changes in blood flow. Initially, the venous and lymphatic outflow are compromised resulting in ovarian edema and enlargement. Later, compromised arterial circulation leads to ischemia and hemorrhagic infarction [3].

In particular, when there is a substantial clinical suspicion of torsion one has to be aware that the absence of a twisted pedicle or abnormal contrast enhancement cannot exclude the diagnosis, unless an appropriate other cause is found [3].

\section{Disclosure}

No conflicts of interest. No grants or financial support.

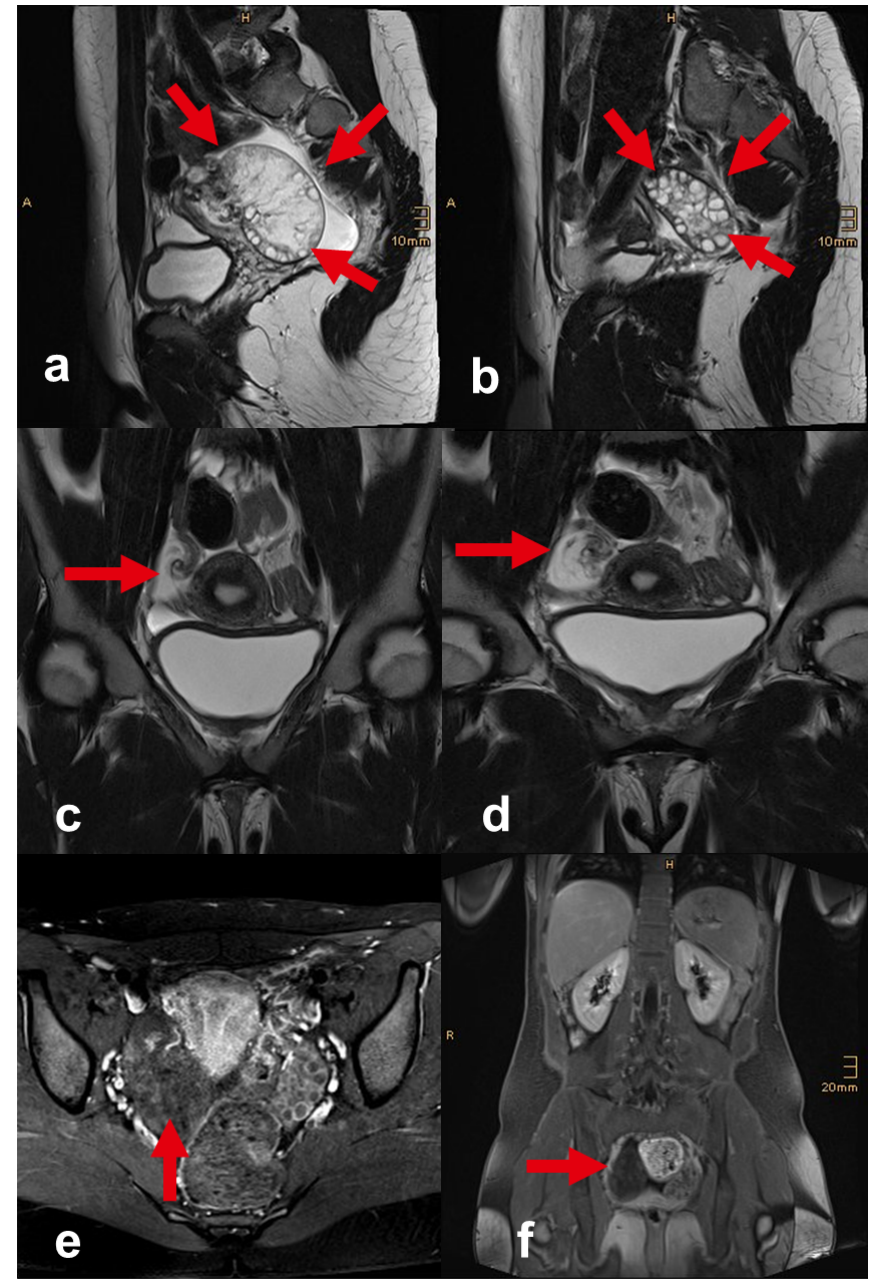

Figure 1. Ovarian torsion. MR imaging: sagittal T2-weighted turbo spin-echo shows edematous right ovary, follicle cysts are typically in peripheral location (a). Normal left ovary on sagittal T2-weighted turbo spin-echo with multiple follicle cysts (b). Coronal T2weighted turbo spin-echo sequence shows an ovarian volvulus on the right side with a twisted vascular pedicle $(c, d)$. Transverse T1-weighted turbo spin-echo with fat saturations after contrast agent administration (e), as well as T1-weighted VIBE after Gadolinium (f) demonstrate, that there is no pathological contrast enhancement.

Correspondence to: Julia Röper-Kelmayr, Central Radiology Institute, Kepler University Hospital, Medical Faculty of the Johannes Kepler University, Linz, Austria, E-mail: julia.roeper-kelmayr@kepleruniklinikum.at

Key words: Ovary, torsion, MR imaging

Received: March 05, 2018; Accepted: March 21, 2018; Published: March 24, 2018 


\section{References}

1. Mandoula C, Verheydena C, Curros-Doyona F, Rathatb G, Taourela P, Milleta I (2017) Diagnostic performance of CT signs for predicting adnexal torsion in women presenting with an adnexal mass and abdominal pain: A case-control study. Eur J Radiol 98: 7581. [Crossref]
2. Ayoob AR, Lee JT (2014) Imaging of common solid organ and bowel torsion in the emergency department. AJR 203: 470-481

3. Duigenan S, Oliva E, Lee SI (2012) Ovarian Torsion: Diagnostic features on CT and MRI with pathologic correlation. AJR 198: 122-131.

Copyright: (C2018 Röper-Kelmayr J. This is an open-access article distributed under the terms of the Creative Commons Attribution License, which permits unrestricted use, distribution, and reproduction in any medium, provided the original author and source are credited. 